\title{
Laser applications in surgery
}

\author{
Beina Azadgoli, Regina Y. Baker
}

Division of Plastic and Reconstructive Surgery, Department of Surgery, Keck School of Medicine, Los Angeles, CA 91011, USA

Contributions: (I) Conception and design: B Azadgoli; (II) Administrative support: None; (III) Provision of study materials or patients: None; (IV) Collection and assembly of data: B Azadgoli; (V) Data analysis and interpretation: None; (VI) Manuscript writing: All authors; (VII) Final approval of manuscript: All authors.

Correspondence to: Dr. Regina Y. Baker, MD. Assistant Professor of Surgery, Division of Plastic and Reconstructive Surgery, Department of Surgery, Keck School of Medicine, 1510 San Pablo St. \#415, Los Angeles, CA 90033, USA. Email: Regina.Baker@med.usc.edu.

\begin{abstract}
In modern medicine, lasers are increasingly utilized for treatment of a variety of pathologies as interest in less invasive treatment modalities intensifies. The physics behind lasers allows the same basic principles to be applied to a multitude of tissue types using slight modifications of the system. Multiple laser systems have been studied within each field of medicine. The term "laser" was combined with "surgery," "ablation," "lithotripsy," "cancer treatment," "tumor ablation," "dermatology," "skin rejuvenation," "lipolysis," "cardiology," "atrial fibrillation (AF)," and "epilepsy" during separate searches in the PubMed database. Original articles that studied the application of laser energy for these conditions were reviewed and included. A review of laser therapy is presented. Laser energy can be safely and effectively used for lithotripsy, for the treatment of various types of cancer, for a multitude of cosmetic and reconstructive procedures, and for the ablation of abnormal conductive pathways. For each of these conditions, management with lasers is comparable to, and potentially superior to, management with more traditional methods.
\end{abstract}

Keywords: Lasers; laser lithotripsy; laser therapy

Submitted Jul 21, 2016. Accepted for publication Nov 23, 2016.

doi: 10.21037/atm.2016.11.51

View this article at: http://dx.doi.org/10.21037/atm.2016.11.51

\section{Introduction}

In 1900, Max Planck discovered that light is released, transferred, and absorbed in specific amounts of energy called quanta, and that this was related to the frequency of the radiation and what he discovered to be Planck's constant (1). Shortly after, Einstein published his work on quantum theory, suggesting that most atoms exist in the ground-energy state $\left(\mathrm{E}_{0}\right)$. These $\mathrm{E}_{0}$ molecules can then be converted to higher energy levels when energy is added to them, and in returning to their ground state, the energy is released spontaneously as photons or electromagnetic (EM) waves. He also discovered that when a photon of the same wavelength collides with an excited atom, the two photons are released concurrently, and therefore have equal frequencies. This idea of "stimulated emission" was years later used in the creation of lasers (2).
Theodore Maiman ultimately created the first "laser" (light amplification by stimulated emission of radiation) by using an electrical source to energize a solid ruby (3). Following this bookmark invention, its many possible indications in medicine were rapidly recognized. As the $\mathrm{CO}_{2}$ laser was known to emit a concentrated ray of light that was easily absorbed by water, it became used to vaporize tissue. The neodymium:yttrium-aluminum-garnet (Nd:YAG) laser created coagulative necrosis within tissue, and the visible light lasers were useful for achieving hemostasis (4) (Table 1). Over time, several different active media have been used to create new lasers, resulting in their utility in a wide range of medical subspecialties. The goal of this review is to provide an overview of the physics behind laser systems, demonstrating how the same basic principles can be applied to various tissue types to accomplish the desired effect, and how this has led to the wide range of clinical applications of lasers. 
Table 1 The various lasers commonly used in medicine along with the wavelength at which they operate, their absorption chromophores, and their clinical applications

\begin{tabular}{llll}
\hline Laser & Wavelength $(\mathrm{nm})$ & Absorption chromophore & Application \\
\hline Ruby & 694 & Pigment, hemoglobin & Dermatology, tattoo removal \\
Nd:YAG & 1,064 & Pigment, proteins & Wide applications \\
Er:YAG & 2,940 & Water & Surgery \\
Diode & $630-980$ & Pigment, water (range) & LLLT, PDT, surgery \\
Argon & $350-514$ & Pigment, hemoglobin & Surgery, PDT, ophthalmology, dermatology \\
$\mathrm{CO}_{2}$ & 10,600 & Water & Surgery \\
Pumped-dye & $504-690$ & Pigment & PDT, dermatology \\
\hline
\end{tabular}

nm, nanometer; LLLT, low level laser therapy; PDT, photodynamic therapy.

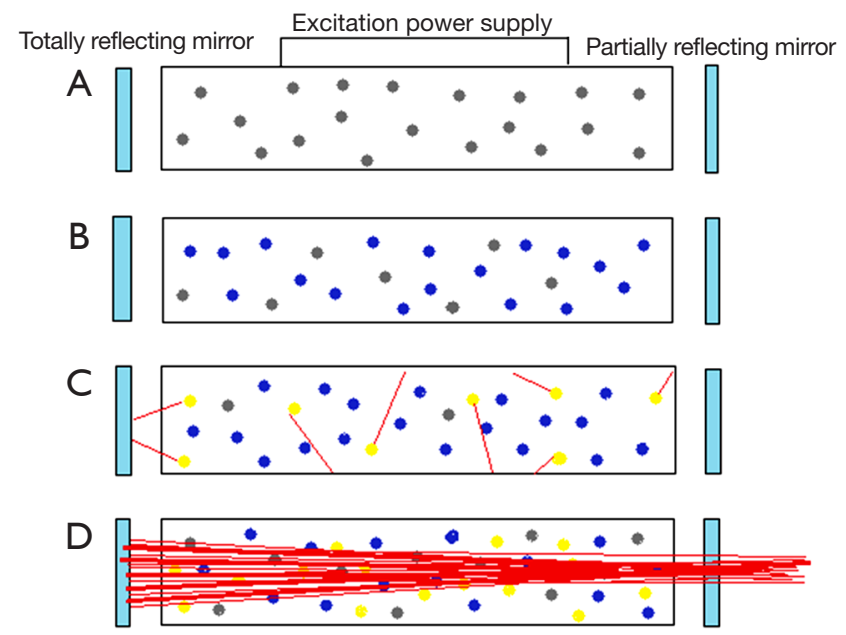

Figure 1 Demonstrates a laser medium at ground state (A) followed by excitation of atoms to higher energy levels (B) and progression to stimulated emission (C) with laser beam generation as a final product (D).

\section{Laser physics}

A simple laser consists of a laser medium (which determines the wavelength of the system) enclosed between two parallel mirrors, one of which is partially reflecting and partially transmitting. The medium is excited by an electrical source until the number of atoms in the excited state is greater than the number in the ground state (population inversion). When the laser medium is activated, it begins to release excited photons spontaneously in all directions. However, a small subset of these photons travels along the centerline of the laser system in a unified fashion between the mirrors.
The mirrors then reflect these photons and the process of stimulated emission is amplified. The partially transmitting mirror then allows a powerful, cohesive beam of photons to be released as laser light (5) (Figure 1).

\section{Laser-tissue interaction}

The effect that a laser has on a sample of tissue is dependent on both properties of the tissue as well as the laser. The tissue properties include its structure, water content, thermal conductivity, heat capacity, density, and its ability to absorb, scatter, or reflect the emitted energy. The properties of the laser that play a role are its power, density, energy content, and wavelength (6).

The main biological targets that are dealt with absorb light very differently, and their optimum absorption spectra depend on the wavelength of the incident photon energy. For the visible light and some near-infrared lasers, the main target chromophores (any substance that absorbs light) are hemoglobin and melanin, whereas for $\mathrm{CO}_{2}$ lasers, the only chromophore is water. In order to achieve selective photothermolysis (using energy at high peak powers and short pulse widths to destroy the intended target alone) without damaging the surrounding tissue, the target tissue must contain chromophores that absorb a specific laser wavelength, and these chromophores should not be found in the surrounding tissue (7).

The $\mathrm{CO}_{2}$, Nd:YAG, and Argon lasers are the lasers most commonly used in medicine and surgery (Table 1). The $\mathrm{CO}_{2}$ laser has carbon dioxide gas as its medium and emits energy at 10,600 nm. Because its chromophore, water, exists everywhere, $\mathrm{CO}_{2}$ lasers cannot be used for selective 
photothermolysis, though they are tissue-selective. All of the incident energy is absorbed in the tissue water down to a specific depth, preventing deeper tissue damage. $\mathrm{CO}_{2}$ lasers operate in the invisible infrared waveband, thus requiring an aiming beam for accurate treatment. Focusing the laser on the tissue produces extremely high power density resulting in instant vaporization and ablation of the tissue. As the irradiance of the laser beam is proportional to the inverse of the square of the diameter of the beam, by defocusing the beam, the surgeon is able to easily change the laser from incision mode to bulk vaporization or coagulation. The $\mathrm{CO}_{2}$ laser has a number of beam modes, each of which reacts differently with the tissue. The simplest mode is continuous wave $(\mathrm{CW})$, in which the laser beam is emitted, operated for a specific time, and then turned off. More recent lasers however are quasi-CW (ultrapulsing), meaning they produce short high-peak power pulses with very long inter-pulse intervals. This has the advantage of allowing more precise incisions with minimal heat build-up because each pulse that is delivered is shorter than the time it takes for the target tissue to cool (7).

The active medium of the Nd:YAG laser is a single YAG crystal bar covered with neodymium ions. The wavelength of light that is produced by of this system, which is determined by the neodymium ions, is $1,060 \mathrm{~nm}(5)$. Because there are no key tissue chromophores at this wavelength, the Nd:YAG laser-tissue interaction produces largely a scattering effect (8). Scattering leads to reflection, which prevents the typical narrow, cohesive beam from being produced. This decreases the penetrative ability of the laser, resulting in slower heating of the tissue (9). This property of the Nd:YAG laser makes it ideal for hemostasis and tumor necrosis, as well as numerous endoscopic procedures within various specialties $(6,10)$.

Ion lasers, such as the argon and krypton laser, operate similarly to gas lasers, except they ionize the active medium. This excites ions instead of atoms, using a large power supply. They can operate at both pulsed and CW modes and can produce wavelengths anywhere between 250 and $530 \mathrm{~nm}$, with the two most powerful beams being in the blue $(488 \mathrm{~nm})$ and green $(514.5 \mathrm{~nm})$ ranges of the spectrum (6).

\section{Clinical applications of lasers}

As minimally invasive techniques are continually being sought out for the treatment of different pathologic processes, the use of lasers has become increasingly popular in modern medicine. In addition to their practical usefulness in the operating room, lasers have a wide range of applications in ophthalmology, lithotripsy, the diagnosis and treatment of various cancers, as well as dermatologic and cosmetic procedures.

\section{Lithotripsy}

Laser lithotripsy has been a widely accepted technique for the fragmentation of urinary and biliary stones for the past few decades (11). Lasers can accomplish lithotripsy by having a photoacoustical/photomechanical effect (laser-induced shockwave lithotripsy) or a predominantly photothermal effect. Of the lasers commonly used in lithotripsy, the 1-psec pulsed-dye laser is the most popular shockwave laser and has been extensively studied (12-14). This device is based on the excitation of coumarin dye to produce the monochromatic light that fragments the calculi (14). At $504 \mathrm{~nm}$, a green light that is absorbed largely by the yellow-colored urinary calculi is produced, which allows it to be safely used without causing much damage to surrounding tissues (13). As the stone absorbs the energy from the laser, the excited ions that are released form a quickly expanding and pulsating cloud around the stone, creating a shock wave that then breaks the calculus into fragments (15). Because this laser is ineffective against the nonabsorbent colorless calculi such as those composed of cystine, photosensitizers (dye) have successfully been used as irrigation fluids and absorbents to initiate the process of fragmentation $(16,17)$. The Q-switched Nd:YAG laser also accomplishes lithotripsy by this mechanism, but it generates larger-magnitude of shockwaves (18).

The long-pulsed Holium:YAG laser on the other hand, uses a mainly photothermal mechanism to fragment calculi (19) (Figure 2A). The laser produces light with a wavelength of $2,100 \mathrm{~nm}$, which is highly absorbable by water. Thus in the appropriate environment, fluid absorbs the energy and is heated as a result. A cloud of vapor is produced, parting the water and allowing the remaining portion of the laser light to directly contact the calculus surface, drilling holes into it and leading to its fragmentation (13). A study conducted by Cimino et al. demonstrated that Ho:YAG laser lithotripsy is a more efficacious endoscopic technique for the treatment of ureteral stones with higher stone fragmentation rates compared to pneumatic lithotripsy, and a review conducted by Teichman concluded that this laser is safe, effective, and works just as well if not better than other modalities, and that it may also be used for biliary stones $(20,21)$. 

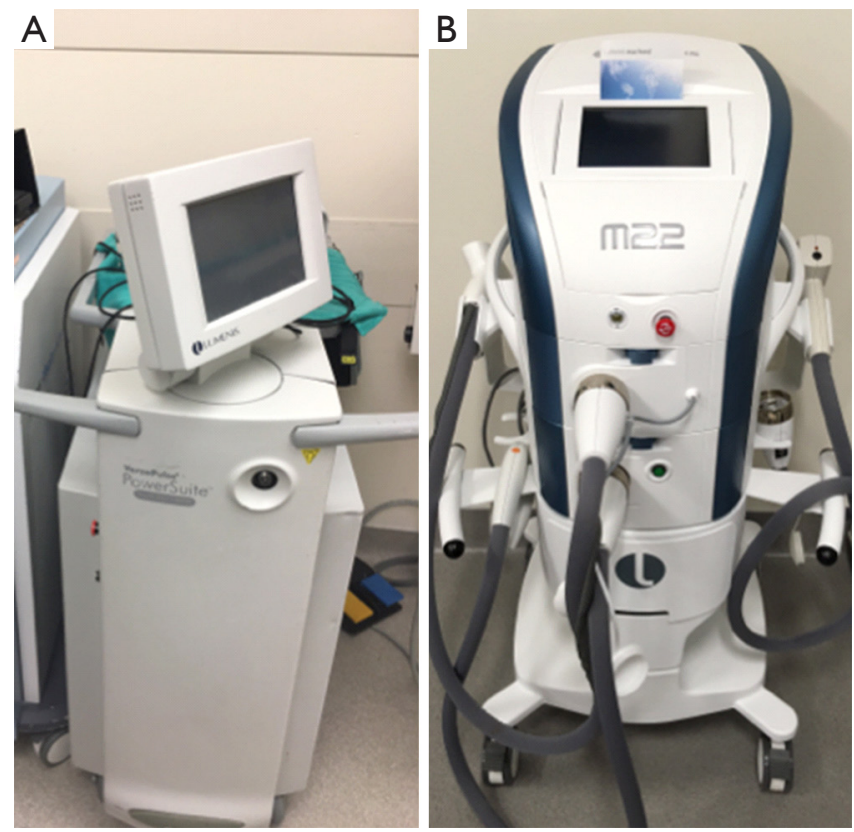

Figure 2 Demonstrates a Ho:YAG lithotripsy laser (A) and an neodymium:yttrium-aluminum-garnet (Nd:YAG) dermatologic laser (B).

\section{Oncology}

Lasers are currently being safely used for the treatment of cancers arising in various organ systems. In neurosurgery for example, laser interstitial thermal therapy (LITT) is a preferred treatment option for patients who are not ideal surgical candidates (22). Since their introduction to neurosurgery, lasers have become increasingly safe to use and have been successfully applied for the treatment of unresectable gliomas as well as hard and hemorrhagic tumors such as meniniomas, tumors of the deep skull base, or tumors deep in the ventricles $(10,23)$. Mucosal ablation techniques using lasers are currently being widely and successfully used for the treatment of superficial gastrointestinal cancers including early gastric cancer, superficial esophageal cancer, colorectal adenoma, and high-grade Barrett's esophagus (24). Moreover, photodynamic therapy (PDT) using lasers has also been shown to be an effective treatment modality for specific types of lung cancer lesions (25).

Direct laser ablation has been used for direct destruction of cancer cells through its photochemical, photomechanical, and photothermal effects. The photochemical reactions that occur ultimately form toxic radicals that lead to the death of tissues, the photomechanical reactions induce stress on the tissue and lead to its fragmentation and the photothermal reactions induce heating and coagulation, which cause cell death (26).

To enhance this process and more accurately target the desired tumor cells, PDT was developed nearly a century ago and has gained great popularity since. This treatment modality involves the administration of a photosensitizing drug followed by the subsequent illumination of the target area with visible light corresponding to the absorbance wavelength of the photosensitizing drug (27). The photosensitizer, which is then activated, initially forms the excited singlet state and then transitions to the triplet state, which in the presence of oxygen form reactive oxygen species that are destructive to neoplastic cells (28). Selective photothermal therapy, on the other hand, uses localized light-absorbing dye to enhance the laser-induced destruction of the tumor cells (29).

\section{Aesthetic and reconstructive surgery}

The unique ability of lasers to target specific structures and layers of tissue makes them a powerful tool in cosmetic and reconstructive surgery. Laser resurfacing has been a major tool used for anti-aging treatment in recent medicine, as the induction of new collagen formation is known to lessen the effects of photoaging (30). Original skin resurfacing techniques involved using ablative $\mathrm{CO}_{2}$ and Er:YAG laser systems to target a specific portion of the dermis. However, because these systems also remove a significant amount of epidermis, they result in prolonged recovery and increased side effects such as infections and erythema. Nonablative lasers, such as the intense pulsed light, Nd:YAG, diode, and Er:glass lasers, which mostly release infrared light, were subsequently developed to overcome these issues (Figure 2B). The goal of these systems is to target the water in the dermis, which during the process heats collagen and induces remodeling. Because there is a system that simultaneously cools the epidermis, tissue evaporation does not occur and no external wound is produced. Most recently, fractionated laser resurfacing has become the basis of skin resurfacing. Using fractionated lasers, fine beams of high-energy light are used to inducing small zones of thermal damage ("microscopic thermal zones") and treating only fractions of skin at a time (31).

Laser-assisted lipolysis, which uses an optical fiber inserted inside a 1-mm cannula, has also become an increasingly popular procedure in cosmetic surgery. Due to the small cannula size, a smaller incision is needed, resulting in less bleeding and scar formation. Of all the lasers that are available 
for medical purposes, those with $920 \mathrm{~nm}$ wavelengths have the smallest absorption coefficient in fat tissue, and so they penetrate the deeper layers of tissue. Those with wavelengths in the 1,320-1,444 $\mathrm{nm}$ range have the largest absorption coefficient in fat, causing smaller penetration depth and allowing for superficial treatment of such tissues. The Nd:YAG laser $(1,064 \mathrm{~nm})$ is the system that is used most widely in laser lipolysis, as the absorption coefficient of fat tissue in this wavelength results in good penetration depth with medium absorption, causing only moderate temperature elevation and thus less tissue damage (32). Further, the coagulation of small blood vessels by the laser light at this wavelength results in significantly less blood loss during the procedure (33). Abdelaal and Aboelatta were able to show a significant decrease in blood loss (54\%) with laser-assisted liposuction when compared to traditional methods (34). Additionally, a review conducted by Mordon and Plot concluded that laser lipolysis produces more even skin results (35).

Finally, the ability of lasers to selectively target pathologic vasculature makes them an ideal source for the treatment of vascular defects such as port-wine stains (36). Prior to use of lasers, patients did not have many treatment options for these types of abnormalities. Currently however, lasers that are preferentially absorbed by hemoglobin over melanin are used for this purpose, with little trauma to the epidermis (37). More recently, lasers with longer wavelengths, and thus the ability to achieve deeper tissue penetration, have also been introduced (38).

\section{Ablation of conductive patbways}

After it was discovered that the pulmonary veins (PV) are an important source of ectopic beats that lead to the paroxysms of atrial fibrillation (AF), the development of catheter ablation devices was inspired for circumferential PV isolation (PVI) (39). Today, the laser balloon catheter is one of the endoscopic ablation systems (EAS) commonly used for the treatment of AF. The device consists of a catheter with a compliant balloon at its tip that is continuously flushed with deuterium oxide. The catheter is introduced into the left atrium and an endoscope is then inserted into the catheter shaft, allowing direct visualization of the ablation target inside the heart. Ablation is performed with a $980-\mathrm{nm}$ diode laser that is housed in the central lumen, emitting laser energy perpendicular to the catheter shaft covering an arc of a $30^{\circ}$ angle and allowing circular ablation around each PV. Laser at this wavelength is not absorbed by deuterium oxide. As a result, it penetrates tissue beyond the endothelium, where it is absorbed by water molecules, resulting in heating and coagulation necrosis. The energy that is delivered can be titrated by changing the power $(5.5-12 \mathrm{~W})$ in a set of predefined levels (40). The energy levels are altered depending on which cardiac wall is being targeted (41). The Nd:YAG laser is another laser system that is commonly used for this purpose (42). A multicenter study conducted by Metzner et al. has shown significant success rates of PVI using EAS, and has suggested that the 1-year success rate is comparable to conventional PVI techniques (about 63\%) (43).

In order to successfully result in a complete conduction block, a fully transmural lesion must be created in the heart. Melby et al. demonstrated that electrical impulses, both paced and AF, could still propagate even through very narrow gaps $(\geq 1 \mathrm{~mm})$ in the ablation line (44). When comparing the effects of different energy levels, studies have shown that the use of higher energy levels results in higher rates of PVI with lower AF recurrence rates and no compromise of the safety profile $(45,46)$.

In neurological surgery, MRI-guided laser-induced thermal therapy (MRgLITT) is commonly used to treat refractory epilepsy, either as a means of ablating the epileptic foci, or as a disconnection tool. MRgLITT combines a diode laser (980-nm) with imaging technology to provide intraoperative information that is necessary for controlling the amount of energy delivered $(47,48)$.

A review conducted by Bandt et al. demonstrated the successful use of laser ablation for the management of refractory epilepsy of many different focal origins including mesial temporal lobe epilepsy, cortical dysplasia, post-stroke neocortical focus, encephalocele, periventricular nodular heterotopia, and hypothalamic hamartomas (48). In addition to resective techniques for epilepsy management, there are disconnective treatment strategies that separate the epileptogenic brain from the nonepileptogenic brain by corpus callosotomy or hemispheretomy. Calistro et al. demonstrated successful endoscopic disconnection of hypothalamic hamartomas with the use of a robot-assisted thulium-laser and Choudhri et al. successfully demonstrated the use of a carbon dioxide laser for corpus callosotomy in children $(49,50)$.

\section{Discussion}

Since their development, the use of lasers in medicine has become extremely widespread and often imperative. From life-threatening diseases to psychologically stressful 
cosmetic defects, laser therapy has led to advancements in countless pathologies, ultimately benefiting both patients and physicians. The evolution of laser technology thus far has led to the practice of minimally invasive procedures, shorter recovery times, and less risk to patient health. As laser technology continues to improve in precision and safety, their applications in medicine are sure to expand to continue providing safer outcomes, more optimal clearance of disease, and improved patient satisfaction.

\section{Acknowledgements}

None.

\section{Footnote}

Conflicts of Interest: The authors have no conflicts of interest to declare.

\section{References}

1. Dicke RH. Coherence in Spontaneous Radiation Processes. Phys Rev 1954;93:99-110.

2. Einstein A. Zur Quantentheorie der Strahlung. Phys Zeitschrift 1917;18:121-8.

3. Maiman TH. Stimulated optical radiation in ruby. Nature 1960;187:493-4.

4. Parker S. Introduction, history of lasers and laser light production. Br Dent J 2007;202:21-31.

5. Fuller TA. The physics of surgical lasers. Lasers Surg Med 1980;1:5-14.

6. Knappe V, Frank F, Rohde E. Principles of lasers and biophotonic effects. Photomed Laser Surg 2004;22:411-7.

7. Omi T, Numano K. The Role of the $\mathrm{CO}_{2}$ Laser and Fractional $\mathrm{CO}_{2}$ Laser in Dermatology. Laser Ther 2014;23:49-60.

8. Edwards MS, Boggan JE, Fuller TA. The laser in neurological surgery. J Neurosurg 1983;59:555-66.

9. Halldórsson T, Rother W, Langeholc J, et al. Theoretical and experimental investigations prove Nd:YAG laser treatment to be safe. Lasers Surg Med 1981;1:253-62.

10. Takeuchi J, Handa H, Taki W, et al. The Nd:YAG laser in neurological surgery. Surg Neurol 1982;18:140-2.

11. Dretler SP. Laser lithotripsy : a review of 20 years of research and clinical applications. Lasers Surg Med 1988;8:341-56.

12. Chan KF, Pfefer TJ, Teichman JM, et al. A perspective on laser lithotripsy: the fragmentation processes. J Endourol
2001;15:257-73.

13. Adams DH. Holmium:YAG laser and pulsed dye laser: a cost comparison. Lasers Surg Med 1997;21:29-31.

14. Grasso M, Bagley D, Sullivan K. Pulsed dye laser lithotripsy--currently applied to urologic and biliary calculi. J Clin Laser Med Surg 1991;9:355-9.

15. Smith Jr JA, Stein BS, Benson Jr RC. Lasers in Urologic Surgery. 3rd ed. St. Louis: Mosby, 1994:192.

16. Ceccheti W, Tasca A, Guazzieri S, et al. Photosensitization method to improve lithotripsy with dye and alexandrite lasers. Proc SPIE 1993;1879:160-4.

17. Tasca A, Cecchetti W, Zattoni F, et al. Photosensitization of Cystine Stones to Induce Laser Lithotripsy. J Urol 1993;149:709-12.

18. Rink K, Delacretaz G, Salathe RP. Fragmentation process of current laser lithotriptors. Lasers Surg Med 1995;16:134-46.

19. Chan KF, Vassar GJ, Pfefer TJ, et al. Holmium:YAG laser lithotripsy: a dominant photothermal ablative mechanism with chemical decomposition of urinary calculi. Lasers Surg Med 1999;25:22-37.

20. Cimino S, Favilla V, Russo GI, et al. Pneumatic lithotripsy versus holmium:YAG laser lithotripsy for the treatment of single ureteral stones: a prospective, single-blinded study. Urol Int 2014;92:468-72.

21. Teichman JM. Laser lithotripsy. Curr Opin Urol 2002;12:305-9.

22. Sherman JH, Hoes K, Marcus J, et al. Neurosurgery for brain tumors: update on recent technical advances. Curr Neurol Neurosci Rep 2011;11:313-9.

23. Hawasli AH, Kim AH, Dunn GP, et al. Stereotactic laser ablation of high-grade gliomas. Neurosurg Focus 2014 Dec;37:E1.

24. Muguruma N, Okamoto K, Timura T, et al. Endoscopic ablation therapy for gastrointestinal superficial neoplasia. Dig Endosc 2012;24:139-49.

25. Ikeda N, Usuda J, Kato H, et al. New aspects of photodynamic therapy for central type early stage lung cancer. Lasers Surg Med 2011;43:749-54.

26. Thomsen S. Pathologic analysis of photothermal and photomechanical effects of laser-tissue interactions. Photochem Photobiol 1991;53:825-35.

27. Mroz P, Yaroslavsky A, Kharkwal GB, et al. Cell death pathways in photodynamic therapy of cancer. Cancers (Basel) 2011;3:2516-39.

28. Abrahamse H, Hamblin MR. New photosensitizers for photodynamic therapy. Biochem J 2016;473:347-64.

29. Chen WR, Adamsc RL, Heaton S, et al. Chromophore- 
enhanced laser-tumor tissue photothermal interaction using an 808-nm diode laser. Cancer Lett 1995;88:15-9.

30. Freedman JR, Greene RM, Green JB. Histologic effects of resurfacing lasers. Facial Plast Surg 2014;30:40-8.

31. Aslam A, Alster TS. Evolution of laser skin resurfacing: from scanning to fractional technology. Dermatol Surg 2014;40:1163-72.

32. Majdabadi A, Abazari M. Study of Interaction of Laser with Tissue Using Monte Carlo Method for 1064nm Neodymium-Doped Yttrium Aluminium Garnet (Nd:YAG) Laser. J Lasers Med Sci 2015;6:22-7.

33. Lukac M, Vizintin Z, Zabkar J, et al. QCW Pulsed Nd:YAG 1064 nm Laser Lipolysis. J Laser Heal Acad 2009; 4:1-12.

34. Abdelaal MM, Aboelatta YA. Comparison of Blood Loss in Laser Lipolysis vs Traditional Liposuction. Aesthet Surg J 2014;34:907-12.

35. Mordon S, Plot E. Laser lipolysis versus traditional liposuction for fat removal. Expert Rev Med Devices 2009;6:677-88.

36. Wu EC, Wong BJ. Lasers and optical technologies in facial plastic surgery. Arch Facial Plast Surg 2008;10:381-90.

37. Kelly KM, Choi B, McFarlane S, et al. Description and Analysis of Treatments for Port-wine Stain Birthmarks. Arch Facial Plast Surg 2005;7:287-94.

38. Faurschou A, Olesen AB, Leonardi-Bee J, et al. Lasers or light sources for treating port-wine stains (Review). Cochrane Database Syst Rev 2011;(11):CD007152.

39. Haïssaguerre M, Jais P, Shah DC, et al. Spontaneous initiation of atrial fibrillation by ectopic beats originating in the pulmonary veins. N Engl J Med 1998;339:659-66.

40. Bordignon S, Julian K, Schulte-hahn B, et al. Endoscopic ablation systems. Expert Rev Med Devices 2013;10:177-83.

41. Metzner A, Wissner E, Lin T, et al. Balloon Devices for

Cite this article as: Azadgoli B, Baker RY. Laser applications in surgery. Ann Transl Med 2016;4(23):452. doi: 10.21037/ atm.2016.11.51
Atrial Fibrillation Therapy. Arrhythm Electrophysiol Rev 2015;4:58-61.

42. Dörschler K, Muuler G. The Role of Laser in Cardiac Surgery. Thorac Cardiovasc Surg 1999;47:385-7.

43. Metzner A, Wissner E, Schmidt B, et al. Acute and LongTerm Clinical Outcome After Endoscopic Pulmonary Vein Isolation: Results from the First Prospective, Multicenter Study. J Cardiovasc Electrophysiol 2013;24:7-13.

44. Melby SJ, Lee AM, Zierer A, et al. Atrial fibrillation propagates through gaps in ablation lines: Implications for ablative treatment of atrial fibrillation. Heart Rhythm 2008;5:1296-301.

45. Metzner A, Wissner E, Schoonderwoerd B, et al. The influence of varying energy settings on efficacy and safety of endoscopic pulmonary vein isolation. Heart Rhythm 2012;9:1380-5.

46. Bordignon S, Chun KR, Gunawardene M, et al. Energy titration strategies with the endoscopic ablation system: lessons from the high-dose vs. low-dose laser ablation study. Europace 2013;15:685-9.

47. Tovar-spinoza Z, Carter D, Ferrone D, et al. The use of MRI-guided laser-induced thermal ablation for epilepsy. Childs Nerv Syst 2013;29:2089-94.

48. Bandt SK, Leuthardt EC. Minimally Invasive Neurosurgery for Epilepsy Using Stereotactic MRI Guidance. Neurosurg Clin N Am 2016;27:51-8.

49. Calisto A, Dorfmüller G, Fohlen M, et al. Endoscopic disconnection of hypothalamic hamartomas: safety and feasibility of robot-assisted, thulium laser-based procedures. J Neurosurg Pediatr 2014;14:563-72.

50. Choudhri O, Lober RM, Camara-Quintana J, et al. Carbon dioxide laser for corpus callosotomy in the pediatric population. J Neurosurg Pediatr 2015;15:321-7. 